\section{Molecular Syndromology}

Mol Syndromol 2018;9:45-51

DOI: $10.1159 / 000479177$
Accepted: May 18, 2017

by M. Schmid

Published online: August 29, 2017

\title{
Two Novel Pathogenic MID1 Variants and Genotype-Phenotype Correlation Reanalysis in X-Linked Opitz G/BBB Syndrome
}

\author{
Nuno Maiaa, c Maria J. Nabais Sáb, c Nataliya Tkachenko ${ }^{b}$ Gabriela Soares $^{b}$ \\ Isabel Marques ${ }^{a, c}$ Bárbara Rodrigues ${ }^{a, c, d}$ Ana M. Fortuna ${ }^{b, c}$ Rosário Santos ${ }^{a, c}$ \\ Arjan P.M. de Brouwer ${ }^{\mathrm{e}}$ Paula Jorge ${ }^{\mathrm{a}, \mathrm{c}}$ \\ a Unidade de Genética Molecular, and b Serviço de Genética Médica, Centro de Genética Médica Doutor Jacinto de \\ Magalhães (CGMJM), Centro Hospitalar do Porto, EPE, and ' Unidade Multidisciplinar de Investigação Biomédica \\ (UMIB), Instituto de Ciências Biomédicas Abel Salazar (ICBAS), Universidade do Porto, Porto, and d Departamento de \\ Biologia, Universidade de Aveiro, Aveiro, Portugal; ' Department of Human Genetics, Donders Institute for Brain, \\ Cognition and Behaviour, Radboud University Nijmegen, Nijmegen, The Netherlands
}

\section{Established Facts}

- To date, 103 index male X-linked Opitz G/BBB syndrome (XLOS) patients with a MID1 pathogenic variant have been identified.

- The major clinical features of XLOS seem to be associated with specific impaired MID1 domains.

\section{Novel Insights}

- Two novel pathogenic MID1 variants were characterized in 4 male XLOS patients.

- No genotype-phenotype correlation could be established between clinical features and the type of MID1 mutations.

- More accurate phenotyping of XLOS patients may disclose potential genotype-phenotype correlations in the future.

\section{Keywords}

Genotype-phenotype correlation $\cdot$ MID1 pathogenic variants $\cdot$ X-linked Opitz G/BBB syndrome

\section{Abstract \\ X-linked Opitz G/BBB syndrome (XLOS) is a multisystemic congenital condition, caused by mutations in the midline-1 gene (MID1), characterized by a large inter- and intrafamilial phenotypic variability and often associated with intellectual}

\section{KARGER}

๑ 2017 S. Karger AG, Basel

E-Mail karger@karger.com

www.karger.com/msy disability (ID). We report clinical, genetic, and molecular findings in 4 patients with typical XLOS dysmorphic features belonging to 2 unrelated families. Two novel pathogenic lossof-function MID1 variants, a maternally inherited c.1656del and a de novo c.1215_1228dup, were identified. Subsequently, we performed a genotype-phenotype analysis using data from 91 male XLOS patients. To test the mutation impact on the phenotype; the type of mutation, the MID1impaired domain and function were compared with the presence of each of the major clinical features (hyper- 
telorism, clefts of the lip and/or palate, laryngo-tracheoesophageal abnormalities, hypospadias and ID ) and minor clinical features (brain, heart, and anal defects). No statistically significant correlation was found with these features. Further investigations, as well as exhaustive and unequivocal phenotyping, may be required to improve our knowledge of the biological mechanisms underlying this syndrome and to provide more adequate disease management.

(c) 2017 S. Karger AG, Basel

Opitz G/BBB syndrome is a multiple midline congenital condition recognizable by minor facial anomalies such as telecanthus/hypertelorism, a broad nasal bridge, and clefts of the lip and/or palate (CLP). Other midline features include congenital heart defect, imperforate anus, hypospadias, malformations of the central nervous system (including hypoplasia of the corpus callosum and agenesis of cerebellar vermis), laryngo-tracheo-esophageal (LTE) defects, and intellectual disability/developmental delay (ID/DD) [Fontanella et al., 2008]. The disorder can be inherited in an X-linked (OMIM 300000) or autosomal dominant (OMIM 145410) manner [Robin et al., 1995; Kruszka et al., 2015]. The X-linked form, Xlinked Opitz G/BBB syndrome (XLOS), is caused by pathogenic variants in the midline-1 gene (MID1 OMIM *300552) located in the short arm of the X chromosome (Xp22.2). MID1 spans $400 \mathrm{~kb}$ and is composed of 10 exons which encode a 667 -amino acid protein belonging to the tripartite motif subfamily of zinc finger proteins [Quaderi et al., 1997]. XLOS shows variable penetrance particularly in female carriers who can be mildly affected, presenting with hypertelorism or, more rarely, other clinical features such as short uvula or lack of an incisor, and CLP. The extensive inter- and intrafamilial phenotypic variability is yet to be explained [Preiksaitiene et al., 2015].

To date, 90 different pathogenic variants in MID1 have been described, including missense and nonsense mutations, small insertions and deletions, splice site alterations, exon deletions and duplications as well as deletions of the complete gene [Quaderi et al., 1997; Gaudenz et al., 1998; Schweiger et al., 1999; Cox et al., 2000; De Falco et al., 2003; Winter et al., 2003; Pinson et al., 2004; So et al., 2005; Cho et al., 2006; Mnayer et al., 2006; Shaw et al., 2006; Ferrentino et al., 2007; Fontanella et al., 2008; Hsieh et al., 2008; Taylor and Aftimos, 2010; Ruiter et al., 2010; Zhang et al., 2011; Hu et al., 2012; Huning et al., 2013; Migliore et al., 2013; Ji et al., 2014; Preiksaitiene et al., 2015]. MID1 consists of the RING finger (RING), zinc-binding B-box-1
(B1) and B-box-2 (B2) domains, followed by a coiled-coil region (CC) and $\mathrm{C}$-terminal subgroup one signature (COS), fibronectin type III repeat (FN3) and PRY-SPRY domains [Quaderi et al., 1997; Du et al., 2014]. Functionally impaired MID1 variants have a lower affinity for microtubules, causing degradation of protein phosphatase 2A (PP2A), a microtubule-associated protein. Controlling PP2A is essential for proper hippocampus development, and PP2A absence seems to have a direct impact on the core-midline phenotype observed in XLOS patients [Trockenbacher et al., 2001; Schweiger and Schneider, 2003]. Furthermore, a protein complex including MID1 is involved in mRNA transport and translation by interacting with several proteins, such as elongation factor I alpha, RACK1, Annexin A2, and proteins of the small ribosomal subunits [Aranda-Orgillés et al., 2008, 2011]. Some authors found associations between impaired MID1 B-box domains and frequent anomalies, or between variants in the FN3 domain and milder phenotypes. However, these results were not confirmed in other recent studies where more patients were included [Li et al., 2015].

We performed a genotype-phenotype analysis by using data from 91 XLOS index patients, aiming to investigate the prevalence of the associated clinical features to assist in establishing a proper prognosis and disease management, and ultimately increase our understanding of the mechanisms of disease.

\section{Case Report}

In family A, patients presented with hypertelorism, telecanthus, and anteverted nostrils. The proband, A-III:3, also had CLP, hypospadias, and swallowing difficulties, necessitating a tracheotomy which was removed at 4 years of age. Furthermore, the boy also showed mild early global DD with no learning difficulties and a normal IQ value at the age of 7 years. His maternal cousins, A-III:1 and A-III:2, showed low-set and dysplastic ears besides hypertelorism, telecanthus, and anteverted nostrils. The cousins had a normal IQ and A-III:1 also had hypospadias. Both mothers had hypertelorism. In family B, the proband, B-II:1, presented with hypertelorism, telecanthus, anteverted nostrils, abnormal ears, inverted nipples, and hypospadias. This boy's psychomotor development and IQ were normal (Fig. 1; Table 1). His mother (I:2) showed normal facial features with no hypertelorism.

\section{Methods and Results}

In 4 XLOS male individuals belonging to 2 unrelated families, 2 novel MID1 variants were characterized by Sanger sequencing analysis of all exons and respective flanking regions of the gene (online suppl. Fig. 1; see www.
46

Mol Syndromol 2018;9:45-51 DOI: $10.1159 / 000479177$
Maia et al. 


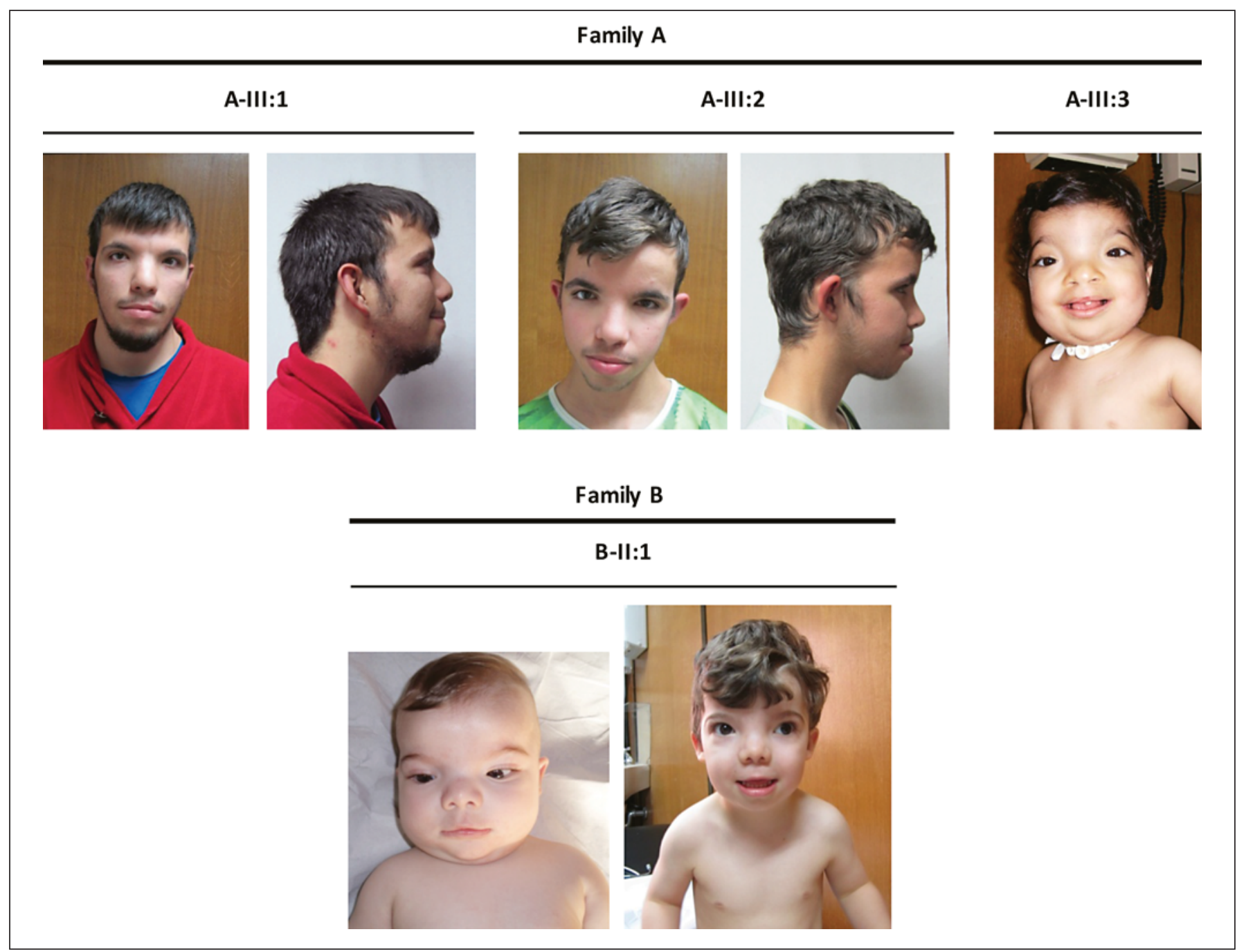

Fig. 1. Craniofacial abnormalities of the XLOS male patients with MID1 pathogenic variants. The 3 patients of family A have hypertelorism, telecantus, and anteverted nostrils. Proband A-III:3 (19 months) shows additional cleft of the lip and palate, and tracheotomy. His maternal cousins (A-III:1, 21 years old and A-III:2, 16 years old) also present with low-set and dysplastic ears. The proband of family B, B-II: 1 (left picture: 6 months old; right picture: 3 years old), has hypertelorism, telecantus, anteverted nostrils, and abnormal ears. karger.com/doi/10.1159/000479177 for all online suppl. material). PCR products were purified using the Illustra ${ }^{\mathrm{TM}}$ ExoStar ${ }^{\mathrm{TM}}$ 1-Step, (GE Healthcare Life Sciences ${ }^{\circledR}$, Little Chalfont, UK) and sequenced using the BigDye ${ }^{\circledR}$ Terminator v3.1 cycle sequencing kit (Applied Biosystems ${ }^{\mathrm{TM}}$, Foster City, CA, USA). Exonic MID1 deletions and duplications were excluded by MLPA analysis (SALSA MLPA P233 MID1 probe mix, MRC-Holland, Amsterdam, The Netherlands). In family A, a frameshift pathogenic variant, c.1656del, p.(Trp552Cysfs*35)/r.(spl?), was identified. This variant occurs at the exon 10 acceptor exonic splice site and presumably affects splicing (GeneSplicer 12.13, MaxEnt

Novel MID1 Variants and GenotypePhenotype Correlation Reanalysis
12.38, HSF 94.37). The proband's maternal cousins, AIII:1 and A-III:2, are hemizygous for the same MID1 pathogenic variant. His mother (II:6) and maternal aunt (II:1) are both heterozygous for the pathogenic variant. Regarding the deceased brothers, one was a stillbirth, and the other brother died at 19 months due to an unclassified "disorder." In both cases, the presence of major anomalies was not mentioned. Both maternal grandfather and grandmother had several brothers and sisters with healthy offspring.

In family $\mathrm{B}$, a frameshift MID1 variant was identified in patient B-II:1: c.1215_1228dup, p.(Ser410Thrfs*34). 
Table 1. Clinical features observed in the male XLOS patients with MID1 pathogenic variants

\begin{tabular}{|c|c|c|c|c|}
\hline & \multicolumn{3}{|l|}{ Family A } & \multirow{2}{*}{$\begin{array}{l}\text { Family B } \\
\text { II:1 (proband) }\end{array}$} \\
\hline & III:3 (proband) & III:1 (cousin) & III:2 (cousin) & \\
\hline Hypertelorism/telecantus & $+/+$ & $+/+$ & $+/+$ & $+/+$ \\
\hline Clefts of the lip/palate & $+/+$ & $-1-$ & $-1-$ & $-1-$ \\
\hline Dysplastic external ears & - & + & + & + \\
\hline Anteverted nostrils & + & + & + & + \\
\hline Laryngo-tracheo-esophageal defects & + & - & + & + \\
\hline Hypospadias & + & + & - & + \\
\hline Anal defects & - & $\begin{array}{l}\text { imperforate and ectopic } \\
\text { anus }\end{array}$ & ectopic anus & - \\
\hline Heart defects & $\begin{array}{l}\text { hypertrophic } \\
\text { cardiomyopathy }\end{array}$ & - & - & - \\
\hline Brain defects & - & - & - & - \\
\hline Intellectual disability & - & - & - & - \\
\hline Additional findings & - & $\begin{array}{l}\text { inguinal and umbilical } \\
\text { hernias } \\
\text { clinodactyly of } 5 \text { th fingers } \\
\text { sindactyly of } 2 \text { nd and } 3 \text { rd } \\
\text { toes }\end{array}$ & chronic constipation & $\begin{array}{l}\text { supranumerary } \\
\text { teeth } \\
\text { inverted nipples } \\
\text { mos } 45, \mathrm{XY}- \\
21[3] / 46, \mathrm{XY}[50]\end{array}$ \\
\hline
\end{tabular}

XLOS, X-linked Opitz G/BBB syndrome; -, absent; +, present.

This genetic alteration was not present in his mother's (I:2) blood or saliva DNA samples (saliva was collected by Oragene ${ }^{\circledR}$ DNA Self-Collection kit, DNA Genotek, Ottawa, ON, Canada). To confirm the correct identification of the samples and their familial relationship, 28 short tandem repeats, AMELX and ZFX genes (Devyser Complete v2 QF-PCR kit, Devyser AB, Hägersten, Sweden) were used (online suppl. Fig. 1). Additionally, in patient B-II:1, karyotype analysis of peripheral blood lymphocytes identified a low-level monosomy of chromosome 21 mosaicism: mos 45,XY,-21[3]/46,XY[50]. No other tissues were available to further assess the mosaicism level. As the clinical descriptions of patients with a mosaic monosomy 21 [Toral-Lopez et al., 2012] are clearly distinct from our patient's clinical outcome and the mosaic mutation occurs only in a low percentage of white blood cells, the contribution of the chromosome 21 monosomy to the phenotype was considered to be minor. Assessment of the X-chromosome inactivation pattern was done by using a Humara assay [Allen et al., 1992] in the peripheral blood DNA of the patient's mother (I:2; online suppl. Fig. 1). In accordance with previously published criteria, $\mathrm{X}$-chromosome inactivation ratios of less than or equal to 80:20 were considered "random," and ratios greater than 90:10 were considered "highly skewed" [Amos-Landgraf et al., 2006]. No significant skewing (>80:20) was detected in any of the 3 samples analyzed.
All variants were described using reference sequence NM_000381.3 following the HGVS recommendations (http://www.hgvs.org/mutnomen/) [den Dunnen and Antonarakis, 2001] and validated using the Mutalyzer (https://mutalyzer.nl/). MID1 transcript information was obtained at Ensembl (http://www.ensembl.org/Homo_ sapiens/Transcript/). MID1 conserved domains were represented according to NCBI Conserved Domain database (http://www.ncbi.nlm.nih.gov/Structure/cdd/cdd. shtml). 1000 Genomes Project, ExAC Browser, ClinVar, and dbSNP databases were used to establish the allele frequency of novel variants. Further prediction of clinical pathogenicity was done by GeneSplicer Web Interface (http://www.cbcb.umd.edu/software/GeneSplicer/gene_ spl.shtml; GeneSplicer threshold $\geq 0$ ), MaxEntScan (http://genes.mit.edu/burgelab/maxent/Xmaxentscan_ scoreseq.html; MaxEnt threshold $\geq 0$ ), and Human Splicing Finder (http://www.umd.be/HSF3/; HSF threshold $\geq 65$ ) to score putative splice sites, and Combined Annotation Dependent Depletion scoring (http://cadd.gs. washington.edu/score; CADD threshold $\geq 15$ ) to score the deleterious effects of single nucleotide variants [Kircher et al., 2014].

\section{Genotype-Phenotype Correlations}

A clear genotype-phenotype correlation would assist in establishing a diagnosis and prognosis as well as disease
48

Mol Syndromol 2018;9:45-51

DOI: $10.1159 / 000479177$
Maia et al. 
management options. Therefore, we performed a review of the previously published 103 index male XLOS patients and described 2 in this study (Fig. 2; online suppl. Table 1). One hundred and five index cases out of 139 XLOS patients were selected for analysis to ensure independent observations. Fourteen cases were excluded from further analyses (for details see online suppl. information). Therefore, only 91 XLOS index patients (80 different variants) were considered. In this subgroup of patients, only 22 had information regarding the complete phenotype. The remaining patients included cases with absent information on: structural brain $(n=68)$, and anal defects $(n=58), \operatorname{ID} / \mathrm{DD}(n=$ $52)$, heart defects $(\mathrm{n}=49)$, LTE defects $(n=37)$, CLP $(n=$ $29)$, hypospadias $(n=17)$, and hypertelorism $(n=13)$. To test the mutation impact on the phenotype, several variables were compared with the presence of each of the major and minor clinical features. The variables tested included the type of mutation, protein domains affected by the mutation, presence of a loss-of-function mutation changing transcript size, and mutations that disrupt protein regions with a known biological role. Association between variables was performed using Pearson $\chi^{2}$ test and Fisher exact test, SPSS for Windows, version 23 (IBM-SPSS, Chicago, IL, USA). A $p$ value of $<0.05$ was considered statistically significant. Correction for multiple testing was performed using the Bonferroni test [Bonferroni, 1935].

The types of MID1 pathogenic variants include premature stop codon $(44 / 92 ; 47.8 \%)$, followed by amino acid changes $(23 / 92 ; 25.0 \%)$, and putatively splicing defects $(13 / 92 ; 14.1 \%)$. XLOS major clinical features (present in $\geq 50 \%$ of the patients) are hypertelorism (89/90;

Fig. 2. Alignment of XLOS patients according to the mutation position along the MID1 functional domains and respective clinical features. MID1 protein domains and gene are represented by rolls and rectangles respectively (not at scale). Each vertical line corresponds to a different index case (see main text for references), and probands described herein are highlighted with a star. Missense mutations are distinguished from the loss of function by vertical black lines. Each clinical feature is symbolized by a colored rectangle, otherwise indicates absence of that feature. Unrelated index cases with the same mutation are connected above, and those with a different mutation affecting the same amino acid are connected below. (a) c.1-?_2004+?del 1-?; (b) c. $-400 \_660+?$ del/c. $-330 \_660+$ ? del; (c) c.-56-?_660+?del; (d) c.1-?_660+?del; (e) c.1-?_660+?dup; (f) c.661-?_756+?del; (g) $\operatorname{arr}(\mathrm{Xp} 22.2)(10,466,103-10,523,151) \times 2$; (h) c.1142_1285del - complex rearrangement of exon 6. B1, zincbinding B-box1; B2, zinc-binding B-box2; CC, a-helicoidal coiledcoil domain; COS, C-terminal subgroup one signature; FN3, fibronectin type III repeat; Rel. Freq, relative frequency (number of index cases with a given clinical feature among those with available data); RING, ring finger motif; PRY, SPRY-associated domain; SPRY, domain in SPla and the RYanodine receptor.

Novel MID1 Variants and Genotype-

Phenotype Correlation Reanalysis

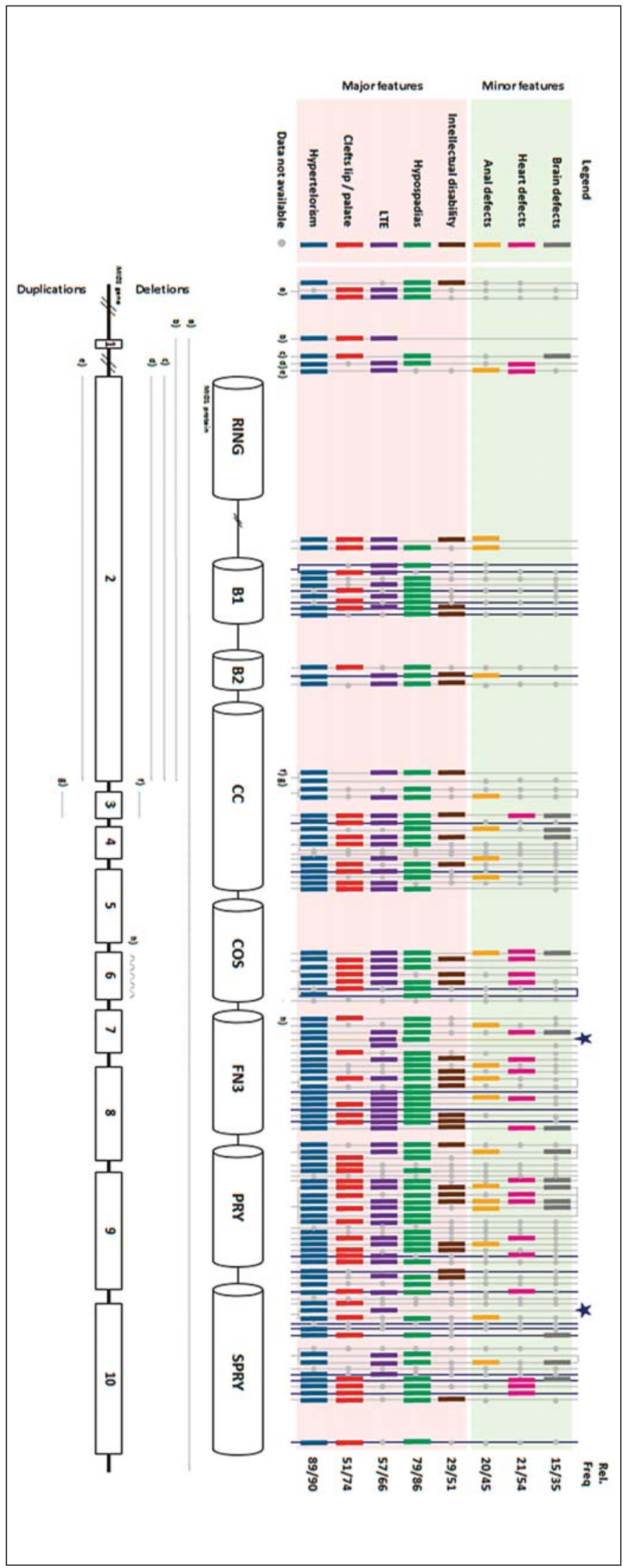

Mol Syndromol 2018;9:45-51 DOI: $10.1159 / 000479177$ 
98.9\%), hypospadias (79/86; 91.9\%), LTE defects (57/66; 86.4\%), CLP (51/74; 68.9\%), and ID/DD (29/51; 56.9\%). Minor clinical characteristics present in $<50 \%$ of the patients include structural anal $(20 / 45 ; 44.4 \%)$, brain $(15 / 35$; $42.9 \%)$, and heart defects $(21 / 54 ; 38.9 \%)$.

We did not observe an association between the presence of any of the clinical features assessed and the affected MID1 domains: RING, B1, B2, CC, COS, FN3, PRY, and SPRY (hypertelorism $p=1.451$, hypospadias $p=1.462$, LTE defects $p=1.558$, CLP $p=1.513$, ID $/ \mathrm{DD}$ $p=1.642$, structural anal $p=0.609$, heart $p=0.256$, and brain defects $p=0.256$ ), nor frequencies of any mutation type (hypertelorism $p=1.451$, hypospadias $p=1.462$, LTE defects $p=1.558$, CLP $p=1.513$, ID $/ \mathrm{DD} p=1.642$, structural anal $p=0.176$, heart $p=0.354$, and brain defects $p=0.193$ ). There was also no association between the frequency of loss-of-function mutations affecting all transcripts and those affecting only longer transcripts in patients with any of the XLOS clinical features (hypertelorism $p=1.624$, hypospadias $p=1.642$, LTE defects $p=1.757, \mathrm{CLP} p=1.730, \mathrm{ID} / \mathrm{DD} p=1.905$, structural anal $p=0.445$, heart $p=0.880$, and brain defects $p=0.653$ ). Finally, we addressed a potential genotype-phenotype association between the 2 MID1 regions with a well-characterized biological role, ubiquitination and microtubules interaction, and XLOS clinical features (hypertelorism $p=1.451$, hypospadias $p=1.462$, LTE defects $p=1.558, \mathrm{CLP} p=0.661, \mathrm{ID} / \mathrm{DD} p=0.609$, structural anal $p=0.741$, brain $p=0.698$, and heart defects $p=0.032$ ). The observed association between heart defects and MID1 function ( $p=0.032)$ was classified as not significant after correction for multiple testing (Bonferronicorrected $p=0.128$ ). Our findings expand the clinical and molecular spectrum of MID1 and do not show any significant genotype-phenotype correlation.

\section{Discussion}

Two novel frameshift pathogenic variants, c.1656del and c.1215_1228dup, presumably resulting in a premature stop codon p.(Trp552Cysfs*35)/r.(spl?) and p.(Ser410Thrfs*34), respectively, were identified in 4 patients. The X-chromosome inactivation patterns in the blood of unaffected carrier females were not skewed, which is in concordance with previously published data [Pinson et al., 2004] and may explain the hypertelorism in carrier mothers (family A: II:1 and II:6). None of the 4 male XLOS patients have a neurodevelopmental or intellectual disabili- ty. Although XLOS is defined as an ID syndrome, this complies with previously reported patients without ID.

Our analyses suggest that there is no correlation between specific major or minor clinical signs and any of the tested variables. This confirms the data of Li et al. [2015]. However, some data due to incomplete phenotyping or relatively poorly described clinical details of patients with XLOS are missing (i.e., full IQ assessment is often missing). Fontanella et al. [2008] suggested an association between truncating mutations (frameshift and nonsense) and the presence of assessed brain deformities (11 out of 12 cases). In the same study, a possible association between CLP abnormalities and mutations affecting splicing of MID1 transcripts was observed. These results could not be confirmed in our study.

Further studies involving more detailed phenotyping in a larger cohort, integrating the data from familial cases, are required to explore the role of specific pathogenic variants in the genesis of this disorder. Therefore, we would argue for a careful assessment of major and minor clinical features of XLOS patients for a better understanding of the molecular role of the protein in the disease phenotype, which may have important clinical and therapeutic implications.

\section{Acknowledgment}

The authors would like to express their gratitude to all patients and families who participated in this research. We are also extremely indebted to the biostatistician Laetitia Teixeira, PhD, (ICBAS) for statistical data analysis and interpretation.

\section{Statement of Ethics}

Participants, or their legal representatives, signed informed consent for the use of DNA samples in research and biobanking as well as permission to publish all images. This study has been approved by the medical ethical committee of the Centro Hospitalar do Porto, EPE - REF 2014.203 (144-DEFI/173-CES). The work was supported by national funds: FCT Strategic Project UID/ Multi/00215/2013 and Centro Hospitalar do Porto, EPE research grants Bolsa de Investigação DEFI 2014 (I.M.). All authors have read the journal's authorship agreement and the manuscript has been reviewed and approved by all authors.

\section{Disclosure Statement}

The authors have no conflicts of interest to declare.

\section{0}

Mol Syndromol 2018;9:45-51 DOI: $10.1159 / 000479177$
Maia et al. 


\section{References}

Allen RC, Zoghbi HY, Moseley AB, Rosenblatt HM, Belmont JW: Methylation of HpaII and HhaI sites near the polymorphic CAG repeat in the human androgen-receptor gene correlates with X chromosome inactivation. Am J Hum Genet 51:1229-1239 (1992).

Amos-Landgraf JM, Cottle A, Plenge RM, Friez $\mathrm{M}$, Schwartz CE, et al: X chromosome-inactivation patterns of 1,005 phenotypically unaffected females. Am J Hum Genet 79:493-499 (2006).

Aranda-Orgillés B, Trockenbacher A, Winter J, Aigner J, Köhler A, et al: The Opitz syndrome gene product MID1 assembles a microtubuleassociated ribonucleoprotein complex. Hum Genet 123:163-176 (2008).

Aranda-Orgillés B, Rutschow D, Zeller R, Karagiannidis AI, Köhler A, et al: Protein phosphatase 2A (PP2A)-specific ubiquitin ligase MID1 is a sequence-dependent regulator of translation efficiency controlling 3-phosphoinositide-dependent protein kinase-1 (PDPK-1). J Biol Chem 286:39945-39957 (2011).

Bonferroni CE: Il calcolo delle assicurazioni su gruppi di teste, in Studi in Onore del Professore Salvatore Ortu Carboni, pp 13-60 (Rome, 1935).

Cho HJ, Shin MY, Ahn KM, Lee SI, Kim HJ, et al: X-linked Opitz G/BBB syndrome: identification of a novel mutation and prenatal diagnosis in a Korean family. J Korean Med Sci 21: 790-793 (2006).

Cox TC, Allen LR, Cox LL, Hopwood B, Goodwin $\mathrm{B}$, et al: New mutations in MID1 provide support for loss of function as the cause of Xlinked Opitz syndrome. Hum Mol Genet 9: 2553-2562 (2000).

De Falco F, Cainarca S, Andolfi G, Ferrentino R, Berti C, et al: X-linked Opitz syndrome: novel mutations in the MID1 gene and redefinition of the clinical spectrum. Am J Med Genet A 120A:222-228 (2003)

den Dunnen JT, Antonarakis SE: Nomenclature for the description of human sequence variations. Hum Genet 109:121-124 (2001).

Du H, Wu K, Didoronkute A, Levy MV, Todi N, et al: MID1 catalyzes the ubiquitination of protein phosphatase $2 \mathrm{~A}$ and mutations within its Bbox1 domain disrupt polyubiquitination of alpha4 but not of PP2Ac. PLoS One 9: e107428 (2014).

Ferrentino R, Bassi MT, Chitayat D, Tabolacci E, Meroni G: MID1 mutation screening in a large cohort of Opitz G/BBB syndrome patients: twenty-nine novel mutations identified. Hum Mutat 28:206-207 (2007).
Fontanella B, Russolillo G, Meroni G: MID1 mutations in patients with X-linked Opitz G/BBB syndrome. Hum Mutat 29:584-594 (2008).

Gaudenz K, Roessler E, Quaderi N, Franco B, Feldman G, et al: Opitz G/BBB Syndrome in Xp22: mutations in the MID1 gene cluster in the carboxy-terminal domain. Am J Hum Genet 63:703-710 (1998).

Hsieh EW, Vargervik K, Slavotinek AM: Clinical and molecular studies of patients with characteristics of Opitz G/BBB syndrome shows a novel MID1 mutation. Am J Med Genet A 146A:2337-2345 (2008).

$\mathrm{Hu} \mathrm{CH}$, Liu YF, Yu JS, Ng YY, Chen SJ, et al: A MID1 gene mutation in a patient with Opitz $\mathrm{G} / \mathrm{BBB}$ syndrome that altered the $3 \mathrm{D}$ structure of SPRY domain. Am J Med Genet A 158A:726-731 (2012)

Huning I, Kutsche K, Rajaei S, Erlandsson A, Lovmar L, et al: Exon 2 duplication of the MID1 gene in a patient with a mild phenotype of Opitz G/BBB syndrome. Eur J Med Genet 56: 188-191 (2013).

Ji X, Xing Y, Xu Y, Liu Y, Chen Y, et al: A novel mutation in $M I D 1$ in a patient with $\mathrm{X}$-linked Opitz G/BBB syndrome. Gene 537:140-142 (2014).

Kircher M, Witten DM, Jain P, O'Roak BJ, Cooper GM, Shendure J: A general framework for estimating the relative pathogenicity of human genetic variants. Nat Genet 46:310-315 (2014)

Kruszka P, Li D, Harr MH, Wilson NR, Swarr D, et al: Mutations in SPECC1L, encoding sperm antigen with calponin homology and coiledcoil domains 1-like, are found in some cases of autosomal dominant Opitz G/BBB syndrome. J Med Genet 52:104-110 (2015).

Li B, Zhou T, Zou Y: Mid1/Mid2 expression in craniofacial development and a literature review of X-linked Opitz syndrome. Mol Genet Genomic Med 4:95-105 (2015).

Migliore C, Athanasakis E, Dahoun S, Wonkam $A$, Lees $M$, et al: Complex rearrangement of the exon 6 genomic region among Opitz G/ BBB Syndrome MID1 alterations. Eur J Med Genet 56:404-410 (2013).

Mnayer L, Khuri S, Merheby HA, Meroni G, Elsas LJ: A structure-function study of MID1 mutations associated with a mild Opitz phenotype. Mol Genet Metab 87:198-203 (2006).
Pinson L, Augé J, Audollent S, Mattéi G, Etchevers $\mathrm{H}$, et al: Embryonic expression of the human MID1 gene and its mutations in Opitz syndrome. J Med Genet 41:381-386 (2004).

Preiksaitiene E, Krasovskaja N, Utkus A, Kasnauskiene J, Meškienè R, et al: R368X mutation in MID1 among recurrent mutations in patients with X-linked Opitz G/BBB syndrome. Clin Dysmorphol 24:7-12 (2015).

Quaderi NA, Schweiger S, Gaudenz K, Franco B, Rugarli EI, et al: Opitz G/BBB syndrome, a defect of midline development, is due to mutations in a new RING finger gene on Xp22. Nat Genet 17:285-291 (1997).

Robin NH, Feldman GJ, Aronson AL, Mitchell HF, Weksberg R, et al: Opitz syndrome is genetically heterogeneous, with one locus on $\mathrm{Xp} 22$, and a second locus on 22q11.2. Nat Genet 11:459-461 (1995).

Ruiter M, Kamsteeg EJ, Meroni G, de Vries BB: A MID1 mutation associated with reduced penetrance of X-linked Opitz G/BBB syndrome. Clin Dysmorphol 19:195-197 (2010).

Schweiger S, Schneider R: The MID1/PP2A complex: a key to the pathogenesis of Opitz BBB/G syndrome. Bioessays 25:356-366 (2003).

Schweiger S, Foerster J, Lehmann T, Suckow V, Muller YA, et al: The Opitz syndrome gene product, MID1, associates with microtubules. Proc Natl Acad Sci USA 96:2794-2799 (1999).

Shaw A, Longman C, Irving M, Splitt M: Neonatal teeth in X-linked Opitz (G/BBB) syndrome. Clin Dysmorphol 15:185-186 (2006).

So J, Suckow V, Kijas Z, Kalscheuer V, Moser B, et al: Mild phenotypes in a series of patients with Opitz GBBB syndrome with MID1 mutations. Am J Med Genet A 132A:1-7 (2005).

Taylor J, Aftimos S: Congenital diaphragmatic hernia is a feature of Opitz G/BBB syndrome. Clin Dysmorphol 19:225-226 (2010).

Toral-Lopez J, Gonzalez-Huerta LM, Cuevas-Covarrubias SA: Complete monosomy mosaic of chromosome 21: case report and review of literature. Gene 510:175-179 (2012).

Trockenbacher A, Suckow V, Foerster J, Winter J, Krauss S, et al: MID1, mutated in Opitz syndrome, encodes an ubiquitin ligase that targets phosphatase $2 \mathrm{~A}$ for degradation. Nat Genet 29:287-294 (2001).

Winter J, Lehmann T, Suckow V, Kijas Z, Kulozik A, et al: Duplication of the MID1 first exon in a patient with Opitz G/BBB syndrome. Hum Genet 112:249-254 (2003).

Zhang X, Chen Y, Zhao S, Markljung E, Nordenskjöld A: Hypospadias associated with hypertelorism, the mildest phenotype of Opitz syndrome. J Hum Genet 56:348-351 (2011).
Novel MID1 Variants and GenotypePhenotype Correlation Reanalysis
Mol Syndromol 2018;9:45-51

DOI: $10.1159 / 000479177$ 